\title{
Identification of the unknown diffusion coefficient in a linear parabolic equation via semigroup approach
}

Ebru Ozbilge ${ }^{* *}$ and Ali Demir ${ }^{2}$

\section{"Correspondence:}

ebru.ozbilge@ieu.edu.tr

'Department of Mathematics,

Faculty of Science and Literature,

Izmir University of Economics,

Sakarya Caddesi, No. 156, Balcova,

Izmir, 35330, Turkey

Full list of author information is

available at the end of the article

\begin{abstract}
This article presents a semigroup approach to the mathematical analysis of the inverse parameter problems of identifying the unknown parameters $p(t)$ and $q$ in the linear parabolic equation $u_{t}(x, t)=u_{x x}+q u_{x}(x, t)+p(t) u(x, t)$, with mixed boundary conditions $u_{x}(0, t)=\psi_{0}, k(1) u(1, t)=\psi_{1}$. The main purpose of this paper is to investigate the distinguishability of the input-output mapping $\Phi[\cdot]: \mathcal{P} \rightarrow H^{1,2}[0, T]$, via semigroup theory. In this paper, it is shown that if the nullspace of the semigroup $T(t)$ consists of only the zero function, then the input-output mapping $\Phi[\cdot]$ has the distinguishability property. It is also shown that both types of boundary conditions and also the region in which the problem is defined play an important role in the distinguishability property of the input-output mapping. Moreover, the input data can be used to determine the unknown parameter $p(t)$ at $(x, t)=(0,0)$ and also the unknown coefficient $q$. Furthermore, it is shown that measured output data $f(t)$ can be determined analytically by an integral representation. Hence the input-output mapping $\Phi[\cdot]: \mathcal{P} \rightarrow H^{1,2}[0, T]$ is given explicitly in terms of the semigroup.
\end{abstract}

\section{Introduction}

The inverse problem of determining parameter in a linear parabolic equation by using over-measured data has generated an increasing interest from engineers and scientist in recent years because such problems play a crucial role in engineering, physics and applied mathematics. The problem of recovering a parameter or parameters in the mathematical model of a physical phenomenon is frequently encountered. Intensive study has been done on these kind of problems and various numerical methods developed which are used to overcome the problem of determining unknown parameter or parameters [14]. Linear identification problems for parabolic equations by the method of semigroups in Hilbert or Banach spaces have been considered by many authors such as Erdogan, Uygun, Sazaklioglu, and Ashyralyev [5-8].

The purpose of this study is to investigate the inverse problem of determining unknown parameters $q$ and $p(t)$ in a one-dimensional parabolic equation using a semigroup approach.

The semigroup approach is an analytical approach for inverse problems of identifying unknown coefficients in parabolic problems. The inverse problem of unknown coefficients in a quasi-linear parabolic equations was studied by Demir and Ozbilge [9-12]. Moreover, the identification of the unknown diffusion coefficient in a linear parabolic equation was 
studied by Demir and Hasanov [12]. The study in this article is based on a philosophy similar to that used in [9-18].

Consider now the following initial-boundary value problem:

$$
\left\{\begin{array}{l}
u_{t}(x, t)=u_{x x}+q u_{x}(x, t)+p(t) u(x, t), \quad(x, t) \in \Omega_{T}, \\
u(x, 0)=g(x), \quad 0<x<1, \\
u_{x}(0, t)=\psi_{0}, \quad k(1) u(1, t)=\psi_{1}, \quad 0<t<T,
\end{array}\right.
$$

where $\Omega_{T}=\left\{(x, t) \in R^{2}: 0<x<1,0<t \leq T\right\}$. The left and right boundary values $\psi_{0}, \psi_{1}$ are assumed to be constants. The functions $p(t)$ and $g(x)$ satisfy the following conditions:

(C1) $p(t) \in H^{0,2}, q \geq 0$;

(C2) $g(x) \in H^{3,2}[0,1], g^{\prime}(0)=\psi_{0}, g(1)=\frac{\psi_{1}}{k(1)}$.

Under these conditions, the initial-boundary value problem (1) has the unique solution $u(x, t) \in H^{2,2}\left(\Omega_{T}\right) \cap H^{3,2}\left(\bar{\Omega}_{T}\right)$.

The parabolic equation in (1) is the mathematical model for various physical and chemical events, such as solute transport in a porous medium, where the dependent variable $u(x, t)$ denotes a solute concentration depending continuously on independent variables $x$ and $t$. The coefficients $q$ and $p(t)$ represent average velocity and decay taking place in both the liquid and absorbed phase. Moreover this linear parabolic equation can be considered as a control problem where the dependent variable $u(x, t)$ represents the temperature. In this case, the parameters $q$ and $p(t)$ can be regarded as control parameters.

Consider the inverse problem of determining the unknown parameters $q$ and $p(t)$ from the Dirichlet type of measured output data at the boundary $x=0$, respectively:

$$
u(0, t)=f(t), \quad t \in(0, T] .
$$

Here $u=u(x, t)$ is the solution of the parabolic problem (1). The function $f(t)$ is assumed to be noisy free measured output data. In this context, parabolic problem (1) will be referred to as a direct (forward) problem, with the inputs $g(x), q$ and $p(t)$. It is assumed that the function $f(t)$ belongs to $H^{0,2}[0, T]$ and satisfies the consistency condition $f(0)=g(0)$.

On denoting $\mathcal{P}:=\left\{(p(t), q): c_{1}>p(t) \geq c_{0}>0, q \geq 0\right\}$, the set of admissible parameters $q$ and $p(t)$, introduce the input-output mapping $\Phi[\cdot, \cdot]: \mathcal{P} \rightarrow C^{1}[0, T]$, where

$$
\Phi[p, q]=\left.u(x, t ; p, q)\right|_{x=0}, \quad(p(t), q) \in \mathcal{P} .
$$

Then the inverse problem with the measured output data $f(t)$ can be formulated as the following operator equation:

$$
\Phi[p, q]=f, \quad(p(t), q) \in \mathcal{P}, f \in H^{0,2}[0, T] .
$$

In this paper, measured output data of Dirichlet type at the boundary $x=0$ is used in the identification of the unknown parameters. Also, in the determination of the unknown parameter, analytical results are obtained.

The purpose of this paper is to study the distinguishability of the unknown parameters via the above input-output mapping. Since the parameter $q$ is a constant, it is relatively easy to determine its exact value in terms of boundary conditions, initial condition and 
over-measured data. Thus, we consider that the mapping $\Phi[\cdot, q]: \mathcal{P} \rightarrow H^{1,2}[0, T]$ has the distinguishability property if $\Phi\left[p_{1}, q\right] \neq \Phi\left[p_{2}, q\right]$ implies $p_{1}(t) \neq p_{2}(t)$. This fact, in particular, shows the injectivity of the inverse mapping $\Phi^{-1}$. In this paper, measured output data of Dirichlet type at the boundary $x=0$ is used in the identification of the unknown parameters. Furthermore, in order to determine the unknown parameter, analytical results are obtained.

The paper is organized as follows. In Section 2, an analysis of the semigroup approach for the inverse problem is given, with the single measured output data $f(t)$ given at the boundary $x=0$. The final section offers some concluding remarks.

\section{An analysis of the inverse problem with given measured data $f(t)$}

Consider now the inverse problem with one measured output data $f(t)$ at $x=0$. In order to formulate the solution of parabolic problem (1) in terms of a semigroup, a new function needs to be defined:

$$
v(x, t)=u(x, t)-\psi_{0} x+\psi_{0}-\frac{\psi_{1}}{k(1)}, \quad x \in[0,1],
$$

which satisfies the following parabolic problem:

$$
\begin{aligned}
& v_{t}(x, t)+A[v(x, t)]=q\left(v_{x}(x, t)+\psi_{0}\right)+p(t)\left(v(x, t)+\psi_{0} x-\psi_{0}+\frac{\psi_{1}}{k(1)}\right), \\
& v(x, 0)=g(x)-\psi_{0} x+\psi_{0}-\frac{\psi_{1}}{k(1)}, \quad 0<x<1, \\
& v_{x}(0, t)=0, \quad k(1) v(1, t)=0, \quad 0<t<T .
\end{aligned}
$$

Here, $A[\cdot]:=-\frac{d^{2}[\cdot]}{d x^{2}}$ is a second order differential operator and its domain is $D_{A}=\{v(x) \in$ $\left.H_{0}^{2,2}(0,1) \cap H_{0}^{3,2}[0,1]: v_{x}(0)=0=v(1)\right\}$, where $H_{0}^{2,2}(0,1)=\overline{C_{0}^{2}(0,1)}$ and $H_{0}^{1,2}[0,1]=\overline{C_{0}^{1}[0,1]}$ are Sobolev spaces. Obviously, by completion, $g(x) \in D_{A}$, since the initial value function $g(x)$ belongs to $C^{3}[0,1]$. Hence $D_{A}$ is dense in $H_{0}^{2,2}[0,1]$, which is a necessary condition for being an infinitesimal generator.

In the following, although the calculations are performed in the smooth function space, by completion they are valid in the Sobolev space.

Denoted by $T(t)$, the semigroup of linear operators are generated by the operator $-A[7$, 8]. Since the differential operator $-A$ is a positive definite and self-adjoint linear operator, the theory of the eigenvalue problems for this operator is well known [19-23].

Note that the eigenvalues and eigenfunctions of the differential operator $A$ can easily be identified. Moreover, the semigroup $T(t)$ can be constructed by using the eigenvalues and eigenfunctions of the infinitesimal generator $A$. Hence, the following eigenvalue problem must first be considered:

$$
\begin{aligned}
& A \phi(x)=\lambda \phi(x), \\
& \phi_{x}(0)=0 ; \quad \phi(1)=0 .
\end{aligned}
$$

This is the Sturm-Liouville problem. The eigenvalues are determined, with $\lambda_{n}=(2 n-$ $1)^{2} \frac{\pi^{2}}{4}$ for all $n=0,1, \ldots$, and the corresponding eigenfunctions are $\phi_{n}(x)=\sqrt{2} \cos ((2 n-$ 
1) $\left.\frac{\pi}{2} x\right)$. In this case, the semigroup $T(t)$ can be represented in the following way [24]:

$$
T(t) U(x, s)=\sum_{n=0}^{\infty}\left\langle\phi_{n}(\theta), U(\theta, s)\right\rangle e^{-\lambda_{n} t} \phi_{n}(x),
$$

where $\left\langle\phi_{n}(\theta), U(\theta, s)\right\rangle=\int_{0}^{1} \phi_{n}(\theta) U(\theta, s) d \theta$. The Sturm-Liouville problem (7) generates a complete orthogonal family of eigenfunctions, so that the null space of the semigroup $T(t)$ is trivial, i.e., $N(T)=\{0\}$. The null space of the semigroup $T(t)$ of the linear operators can be defined as follows:

$$
N(T)=\left\{U(\theta, t):\left\langle\phi_{n}(\theta), U(\theta, t)\right\rangle=0, \text { for all } n=0,1,2,3, \ldots\right\} .
$$

The unique solution of the initial-boundary value problem (6) in terms of semigroup $T(t)$ can be represented in the following form:

$$
v(x, t)=T(t) v(x, 0)+\int_{0}^{t} T(t-s)\left(q\left(v_{x}(x, s)+\psi_{0}\right)+p(s)\left(v(x, s)+\psi_{0} x-\psi_{0}+\psi_{1} / k(1)\right)\right) d s .
$$

Hence, by using identity (5) and taking the initial value $u(x, 0)=g(x)$ into account, the solution $u(x, t)$ of the parabolic problem (1) in terms of semigroup can be written in the following form:

$$
\begin{aligned}
u(x, t)= & \psi_{0} x-\psi_{0}+\frac{\psi_{1}}{k(1)}+T(t)\left(g(x)-\psi_{0} x+\psi_{0}-\frac{\psi_{1}}{k(1)}\right) \\
& +\int_{0}^{t} T(t-s)\left(q u_{x}(x, s)+p(s) u(x, s)\right) d s .
\end{aligned}
$$

In order to arrange the above solution representation, let us define the following:

$$
\begin{aligned}
& \zeta(x)=\left(g(x)-\psi_{0} x+\psi_{0}-\frac{\psi_{1}}{k(1)}\right), \\
& \xi(x, t)=\left(q u_{x}(x, s)+p(s) u(x, s)\right) .
\end{aligned}
$$

The solution representation in terms of $\zeta(x)$ and $\xi(x, s)$ can then be rewritten in the following form:

$$
u(x, t)=\psi_{0} x-\psi_{0}+\frac{\psi_{1}}{k(1)}+T(t) \zeta(x)+\int_{0}^{t} T(t-s) \xi(x, s) d s .
$$

Substituting $x=0$ into this solution yields

$$
u(0, t)=-\psi_{0}+\frac{\psi_{1}}{k(1)}+T(t) \zeta(0)+\int_{0}^{t} T(t-s) \xi(0, s) d s
$$

Taking into account the over-measured data $u(0, t)=f(t)$

$$
f(t)=-\psi_{0}+\frac{\psi_{1}}{k(1)}+T(t) \zeta(0)+\int_{0}^{t} T(t-s) \xi(0, s) d s
$$


is obtained, which implies that $f(t)$ can be determined analytically. The right-hand side of identity (10) defines the semigroup representation of the input-output mapping $\Phi[p]$ on the set of admissible source functions $\mathcal{P}$ :

$$
\Phi[p, q](t):=-\psi_{0}+\frac{\psi_{1}}{k(1)}+T(t) \zeta(0)+\int_{0}^{t} T(t-s) \xi(0, s) d s, \quad \forall t \in[0, T] .
$$

Now, differentiating both sides of identity (8) with respect to $t$ and using the definitions for $\zeta(x)$ and $\xi(x, t)$ yields

$$
\begin{aligned}
u_{t}(x, t)= & T(t) A\left(g(x)-\psi_{0} x+\psi_{0}-\frac{\psi_{1}}{k(1)}\right)+q u_{x}(x, t)+p(t) u(x, t) \\
& +\int_{0}^{t} A T(t-s)\left(q u_{x}(x, s)+p(s) u(x, s)\right) d s .
\end{aligned}
$$

Using the semigroup property $\int_{0}^{t} A T(t-s) u(x, s) d s=T(t) u(x, 0)-T(0) u(x, t)$ where $T(0)=I$ is the identity operator and substituting $t=0$ in (11) we get

$$
u_{t}(x, 0)=g^{\prime \prime}(x)+\left(q u_{x}(x, 0)+p(0) u(x, 0)\right) .
$$

Substituting $x=0$ into (12),

$$
u_{t}(0,0)=g^{\prime \prime}(0)+q u_{x}(0,0)+p(0) u(0,0)
$$

is obtained. Since $u(0, t)=f(t)$, we have $u_{t}(0, t)=f^{\prime}(t)$. Taking this into account and substituting $t=0$ yields

$$
f^{\prime}(0)=g^{\prime \prime}(0)+q u_{x}(0,0)+p(0) u(0,0) .
$$

Solving the equation for $p(0)$, the following explicit formula is obtained:

$$
p(0)=\frac{f^{\prime}(0)-g^{\prime \prime}(0)-g u_{x}(0,0)}{u(0,0)}=\frac{f^{\prime}(0)-g^{\prime \prime}(0)-g \psi_{0}}{g(0)} .
$$

This result implies that $g(0) \neq 0$. Now, taking the derivative of (12) with respect to $x$, we have

$$
u_{x t}(x, 0)=g^{\prime \prime \prime}(x)+\left(q u_{x x}(x, 0)+p(0) u_{x}(x, 0)\right)
$$

Substituting $x=0$ into (14),

$$
u_{x t}(0,0)=g^{\prime \prime \prime}(0)+\left(q u_{x x}(0,0)+p(0) u_{x}(0,0)\right) .
$$

Since $u_{x}(0, t)=\psi_{0}, u_{x t}(0,0)=0$. Solving the equation for $q$, the following explicit formula is obtained:

$$
q=\frac{-g^{\prime \prime \prime}(0)-p(0) u_{x}(0,0)}{u_{x x}(0,0)} .
$$


Substituting (13) in (15), we get

$$
q=\frac{-g^{\prime \prime \prime}(0) g(0)-\left(f^{\prime}(0)-g^{\prime \prime}(0)-g \psi_{0}\right) u_{x}(0,0)}{g(0) u_{x x}(0,0)}
$$

The admissible set of diffusion parameters can then be redefined as follows:

$$
\begin{aligned}
& \mathcal{P}_{q}:=\left\{(p(t), q) \in \mathcal{P}: p(0)=\frac{f^{\prime}(0)-g^{\prime \prime}(0)-g \psi_{0}}{g(0)},\right. \\
&\left.q=\frac{-g^{\prime \prime \prime}(0) g(0)-\left(f^{\prime}(0)-g^{\prime \prime}(0)-g \psi_{0}\right) u_{x}(0,0)}{g(0) u_{x x}(0,0)}\right\} .
\end{aligned}
$$

The following lemma implies the relation between the parameters $p_{1}(t), p_{2}(t) \in \mathcal{P}_{q}$ at $x=0$ and the corresponding outputs $f_{j}(t):=u\left(0, t ; p_{j}\right), j=1,2$.

Lemma 1 Let $u_{1}(x, t)=u\left(x, t ; p_{1}\right)$ and $u_{2}(x, t)=u\left(x, t ; p_{2}\right)$ be the solutions of the direct problem (1), corresponding to the admissible parameters $p_{1}(t), p_{2}(t) \in \mathcal{P}_{q}$. If $f_{j}(t)=u\left(0, t ; p_{j}\right)$, $j=1,2$, are the corresponding outputs, and we denote $\Delta f(t)=f_{1}(t)-f_{2}(t), \Delta \xi(x, t, s)=$ $\xi^{1}(x, t, s)-\xi^{2}(x, t, s)$, then the outputs $f_{j}(t), j=1,2$, satisfy the following integral identity:

$$
\Delta f(\tau)=\int_{0}^{\tau} \Delta \xi(0, \tau-s, s) d s
$$

for each $\tau \in(0, T]$.

Proof By using identity (10), the measured output data $f_{j}(t):=u\left(0, t ; p_{j}\right), j=1,2$ can be written as follows:

$$
\begin{aligned}
& f_{1}(\tau)=-\psi_{0}+\frac{\psi_{1}}{k(1)}+\zeta^{1}(0, \tau)+\int_{0}^{\tau} \xi^{1}(0, \tau-s, s) d s \\
& f_{2}(\tau)=-\psi_{0}+\frac{\psi_{1}}{k(1)}+\zeta^{1}(0, \tau)+\int_{0}^{\tau} \xi^{2}(0, \tau-s, s) d s
\end{aligned}
$$

respectively. From identity (9) it is obvious that $\xi^{1}(0, \tau)=\xi^{2}(0, \tau)$ for each $\tau \in(0, T]$. Hence, the difference between these formulas implies the desired result.

The above lemma and the definition of $\xi(x, t, s)$ enable us to reach the following conclusion:

Corollary 1 Let the conditions of Lemma 1 hold. If, in addition,

$$
\begin{aligned}
& \left\langle\phi_{n}(x), \xi^{1}(x, t)-\xi^{2}(x, t)\right\rangle \\
& =\left\langle\phi_{n}(x), q\left(u_{1}\right)_{x}\left(x, t ; p_{1}\right)-q\left(u_{2}\right)_{x}\left(x, t ; p_{1}\right)+p_{1}(t) u_{1}\left(x, t ; p_{1}\right)-p_{2}(t) u_{2}\left(x, t ; p_{2}\right)\right\rangle=0, \\
& \quad \forall t \in(0, T], n=0,1, \ldots
\end{aligned}
$$

holds, then $f_{1}(t)=f_{2}(t), \forall t \in[0, T]$.

Note that $\left\langle\phi_{n}(x), \xi^{1}(x, t)-\xi^{2}(x, t)\right\rangle=0$ for all $\phi_{n}(x)$ implies that $\xi^{1}(x, \tau)=\xi^{2}(x, \tau)$. Hence, by Lemma 1 , we conclude that $f_{1}(t)=f_{2}(t), \forall t \in[0, T]$. Moreover, this leads to the impor- 
tant conclusion that the input-output mapping $\Phi[p]$ is distinguishable [5], i.e.,

$$
p_{1}(t) \neq p_{2}(t), \quad \Phi\left[p_{1}\right] \neq \Phi\left[p_{2}\right] .
$$

Theorem 1 Let conditions (C1), (C2) hold. Assume that $\Phi[\cdot]: \mathcal{P} \rightarrow H^{1,2}[0, T]$ is the inputoutput mapping defined by (3) and corresponding to the measured output $f(t):=u_{x}(0, t)$. In this case, the mapping $\Phi[p]$ has the distinguishability property in the class of admissible parameters $\mathcal{P}_{q}$, i.e.,

$$
\Phi\left[p_{1}\right] \neq \Phi\left[p_{2}\right], \quad \forall p_{1}, p_{2} \in \mathcal{P}_{q}, \quad p_{1}(t) \neq p_{2}(t) .
$$

\section{Conclusion}

The aim of this study was to analyze the distinguishability properties of the input-output mapping $\Phi[\cdot]: \mathcal{P} \rightarrow H^{1,2}[0, T]$, which is determined by the measured output data. In this study, we conclude that the semigroup with a trivial null space, i.e., $N(T)=\{0\}$, plays a crucial role in the distinguishability of the input-output mappings. The other important conclusion is that for this kind of inverse problem, one Dirichlet type of measured output data at the boundary $x=0$ is sufficient to investigate the distinguishability of the inputoutput mapping, and also we were able to define the value of the unknown function $p(x)$ at $x=0$. Similarly, if we take Neumann type of measured output data, we would find the value of $p(x)$ at $x=1$.

\section{Competing interests}

The authors declare that they have no competing interests.

\section{Authors' contributions}

All authors contributed equally and significantly in writing this paper. All authors read and approved the final manuscript.

\section{Author details}

'Department of Mathematics, Faculty of Science and Literature, Izmir University of Economics, Sakarya Caddesi, No. 156, Balcova, Izmir, 35330, Turkey. ${ }^{2}$ Department of Mathematics, Kocaeli University, Umuttepe, Izmit, Kocaeli 41380, Turkey.

\section{Acknowledgements}

The research was supported by parts by the Scientific and Technical Research Council (TUBITAK) of Turkey and Izmir University of Economics.

Received: 30 September 2013 Accepted: 13 January 2014 Published: 30 Jan 2014

\section{References}

1. DuChateau, P: Monotonicity and invertibility of coefficient-to-data mappings for parabolic inverse problems. SIAM J. Math. Anal. 26, 1473-1487 (1995)

2. Isakov, V: On uniqueness in inverse problems for quasilinear parabolic equations. Arch. Ration. Mech. Anal. 124, 1-13 (1993)

3. Pilant, MS, Rundell, W: A uniqueness theorem for conductivity from overspecified boundary data. J. Math. Anal. Appl. 136, 20-28 (1988)

4. Renardy, M, Rogers, R: An Introduction to Partial Differential Equations. Springer, New York (2004)

5. Ashyralyev, A, San, ME: An approximation of semigroup method for stochastic parabolic equations. Abstr. Appl. Anal. 2012, Article ID 684248 (2012)

6. Erdogan, A, Sazaklioglu, A: A note on the numerical solution of an identification problem for observing two-phase flow in capillaries. Math. Methods Appl. Sci. (2013). doi:10.1002/mma.2985

7. Erdogan, A, Uygun, $\mathrm{H}$ : A note on the inverse problem for a fractional parabolic equation. Abstr. Appl. Anal. 2012, Article ID 276080 (2012)

8. Erdogan, A, Ashyralyev, A: On the second order implicit difference schemes for a right hand side identification problem. Appl. Math. Comput. 226, 212-229 (2014)

9. Demir, A, Ozbilge, E: Semigroup approach for identification of the unknown diffusion coefficient in a quasi-linear parabolic equation. Math. Methods Appl. Sci. 30, 1283-1294 (2007)

10. Ozbilge, E: Identification of the unknown diffusion coefficient in a quasi-linear parabolic equation by semigroup approach with mixed boundary conditions. Math. Methods Appl. Sci. 31, 1333-1344 (2008) 
11. Demir, A, Ozbilge, E: Analysis of a semigroup approach in the inverse problem of identifying an unknown coefficient. Math. Methods Appl. Sci. 31, 1635-1645 (2008)

12. Demir, A, Hasanov, A: Identification of the unknown diffusion coefficient in a linear parabolic equation by the semigroup approach. J. Math. Anal. Appl. 340, 5-15 (2008)

13. Ozbilge, E: Identification of the unknown diffusion coefficient in a quasi-linear parabolic equation by semigroup approach with mixed boundary conditions. Math. Methods Appl. Sci. 31, 1333-1344 (2008)

14. Ozbilge, E, Demir, A: Analysis of a semigroup approach in the inverse problem of identifying an unknown parameters. Appl. Math. Comput. 218, 965-969 (2011)

15. Demir, A, Ozbilge, E: Identification of the unknown boundary condition in a linear parabolic equation. J. Inequal. Appl. 2013, 96 (2013). doi:10.1186/1029-242X-2013-96

16. Ozbilge, E, Demir, A: Identification of the unknown coefficient in a quasi-linear parabolic equation by semigroup approach. J. Inequal. Appl. 2013, 212 (2013). doi:10.1186/1029-242X-2013-212

17. Ozbilge, E: Determination of the unknown boundary condition of the inverse parabolic problems via semigroup method. Bound. Value Probl. 2013, 2 (2013). doi:10.1186/1687-2770-2013-2

18. Ozbilge, E, Demir, A: Semigroup approach for identification of the unknown diffusion coefficient in a linear parabolic equation with mixed output data. Bound. Value Probl. 2013, 43 (2013). doi:10.1186/1687-2770-2013-43

19. Cannon, JR: The One-Dimensional Heat Equation. Addison-Wesley, Reading (1984)

20. DuChateau, P, Thelwell, R, Butters, G: Analysis of an adjoint problem approach to the identification of an unknown diffusion coefficient. Inverse Probl. 20, 601-625 (2004)

21. Showalter, R: Monotone Operators in Banach Spaces and Nonlinear Partial Differential Equations. Am. Math. Soc., Providence (1997)

22. Hasanov, A, Demir, A, Erdem, A: Monotonicity of input-output mappings in inverse coefficient and source problem for parabolic equations. J. Math. Anal. Appl. 335, 1434-1451 (2007)

23. Hasanov, A, DuChateau, P, Pektas, B: An adjoint approach and coarse-fine mesh method for identification of the diffusion coefficient in a linear parabolic equations. J. Inverse III-Posed Probl. 14, 435-463 (2006)

24. DuChateau, P, Gottlieb, J: Introduction to Inverse Problems in Partial Differential Equations for Engineers, Physicists and Mathematicians. Kluwer Academic, Dordrecht (1996)

10.1186/1687-1847-2014-47

Cite this article as: Ozbilge and Demir: Identification of the unknown diffusion coefficient in a linear parabolic equation via semigroup approach. Advances in Difference Equations 2014, 2014:47

\section{Submit your manuscript to a SpringerOpen ${ }^{\circ}$ journal and benefit from:}

- Convenient online submission

- Rigorous peer review

- Immediate publication on acceptance

- Open access: articles freely available online

- High visibility within the field

- Retaining the copyright to your article 